\title{
Metastatic Adrenocortical Carcinoma: A Case Report
}

Annalisa Montebello ${ }^{1}$, Ruth Caruana ${ }^{1,2} \&$ Sandro Vella ${ }^{1,2}$

${ }^{1}$ Department of Medicine, Mater Dei Hospital, Malta; ${ }^{2}$ Department of Medicine, University of Malta Medical School, Malta.

\section{IF}

\section{Introduction}

- Adrenocortical carcinomas (ACC) are rare malignant tumours. An incidence of 1 to 2 per million per year is reported. We present a case of newly diagnosed metastatic ACC.

\section{Case Report}

- 70 yr old lady was admitted with a one month history of new onset hypertension, hypergylcaemia, rapidly progressive hirsutism and generalised weakness.

- O/E: Cushingoid appearance with facial plethora, severe hirsutism, central obesity and severe proximal myopathy.

- CT trunk: a large, lobulated, inhomogeneous, solid left adrenal mass $8 \times 5 \mathrm{~cm}$ in size with enlarged local and paraortic lymph nodes. Pulmonary metastases were noted.

- She was diagnosed with metastatic adrenocortical carcinoma secreting cortisol and androgens. Aldosterone rennin ratio, plasma metanephrines and catecholamines were normal.

- A few days later she complained of severe abdominal pain and was diagnosed with sigmoid bowel perforation needing emergency laparotomy.

- Post operatively she developed severe hypokalaemia of $(1.94 \mathrm{mmol} / \mathrm{L}[3.5-5 \mathrm{mmol} / \mathrm{L}])$ which was difficult to manage with oral potassium supplementation and aldosterone antagonist treatment.

- She became dependent on continuous intravenous potassium replacement therapy.

- Her post operative course was complicated by abdominal wound dehiscence. Wound healing was unsuccessful despite treatment with multiple antibiotic therapy. She additionally developed a thrombosis of the right femoral vein.

-She was given one shot of $50 \%$ doxorubicin-etoposide and cisplatin based chemotherapy. Unfortunately this resulted on neutropenic sepsis which needed treatment with antibiotics and granulocyte colony stimulating factor.

- Her wounds did not heal and she passed away a few weeks later.

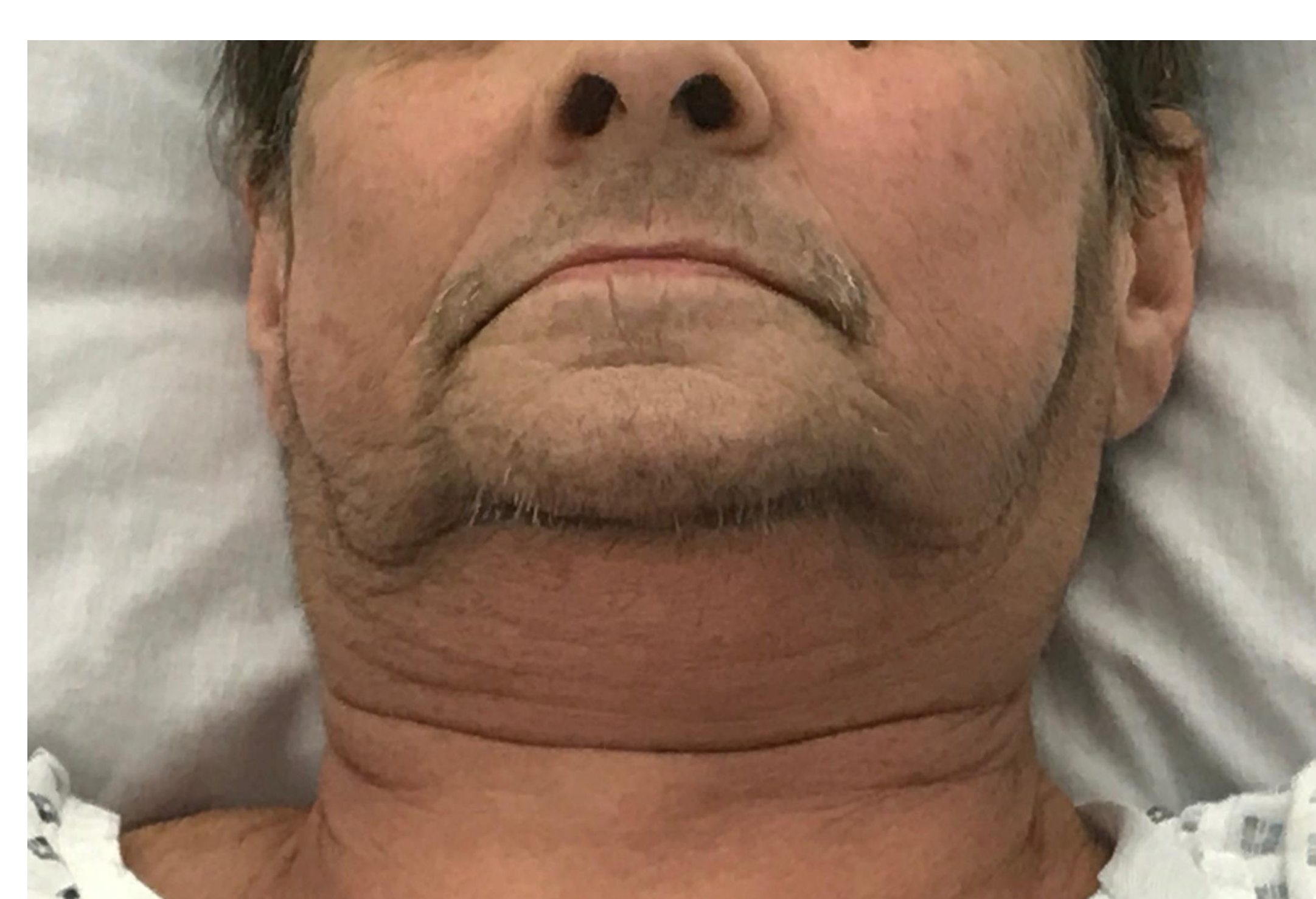

Figure 1: Cushingoid appearance: moon face, facial plethora and severe hirsutism

\begin{tabular}{|l|c|c|}
\hline \multicolumn{1}{|c|}{ Hormone } & Result & Range \\
\hline Random Cortisol & $1209 \mathrm{nmol} / \mathrm{L}$ & $145-619 \mathrm{nmol} / \mathrm{L}$ \\
\hline ACTH & $<5 \mathrm{pg} / \mathrm{mL}$ & $10-48 \mathrm{pg} / \mathrm{mL}$ \\
\hline Total testosterone & $46 \mathrm{nmol} / \mathrm{L}$ & $\mathrm{ND}-1.49 \mathrm{nmol} / \mathrm{L}$ \\
\hline Oestradiol & $507 \mathrm{pmol} / \mathrm{L}$ & $\mathrm{ND}-118 \mathrm{nmol} / \mathrm{L}$ \\
\hline Progesterone & $5.15 \mathrm{nmol} / \mathrm{L}$ & $\mathrm{ND}-3.2 \mathrm{nmol} / \mathrm{L}$ \\
\hline 17 OH progesterone & $21.6 \mathrm{ng} / \mathrm{mL}$ & $0.13-0.6 \mathrm{ng} / \mathrm{mL}$ \\
\hline Androstenedione & $19.4 \mathrm{ng} / \mathrm{mL}$ & $0.35-2.49 \mathrm{ng} / \mathrm{mL}$ \\
\hline DHEAS & $23.3 \mathrm{umol} / \mathrm{L}$ & $0.95-11.67 \mathrm{umol} / \mathrm{L}$ \\
\hline
\end{tabular}

Figure 2: Biochemistry shows a cortisol and androgen co secreting tumour

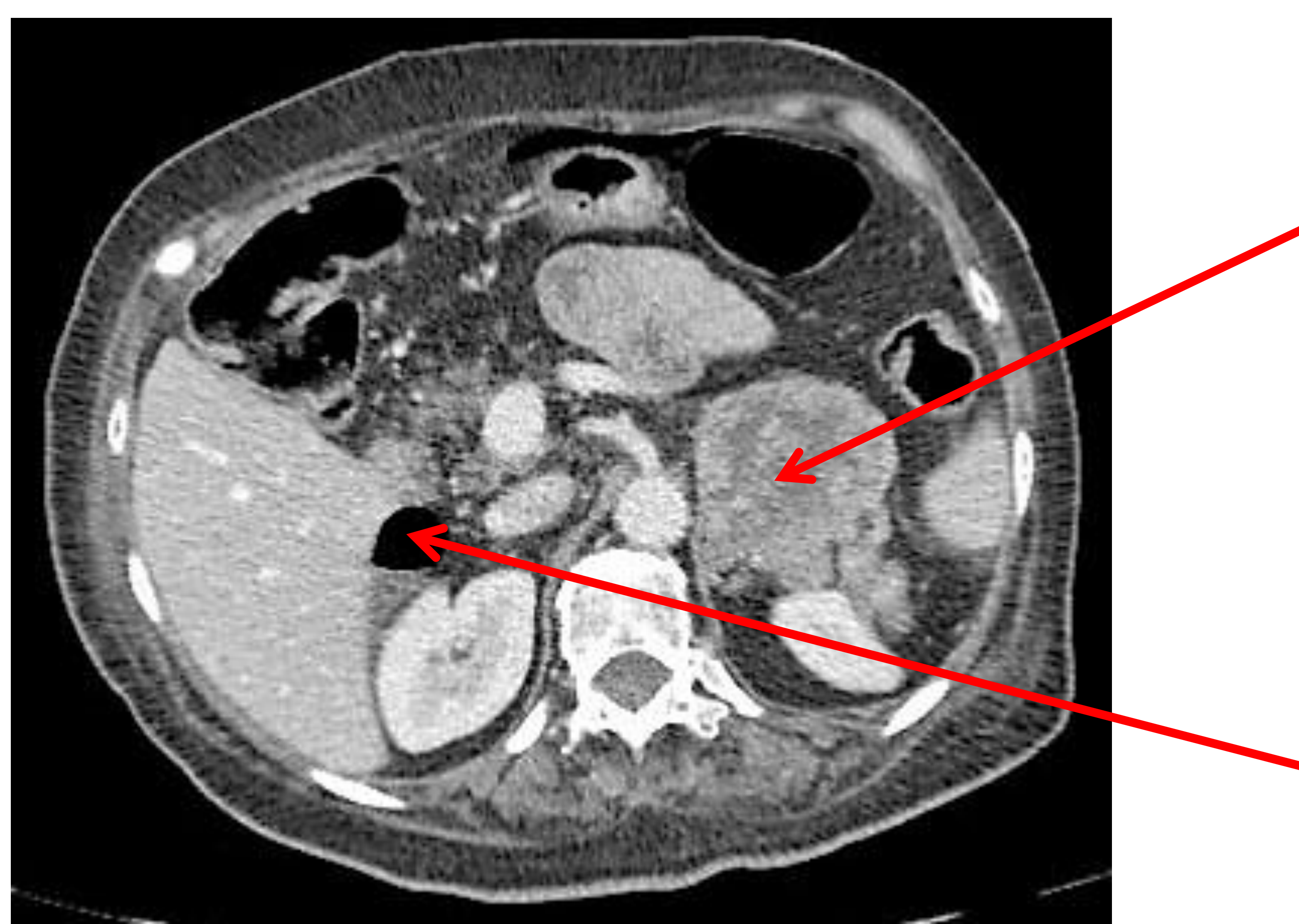

$8 \times 5 \mathrm{~cm}$

Adrenocortical carcinoma

Air in the hepatorenal space due to perforated sigmoid colon

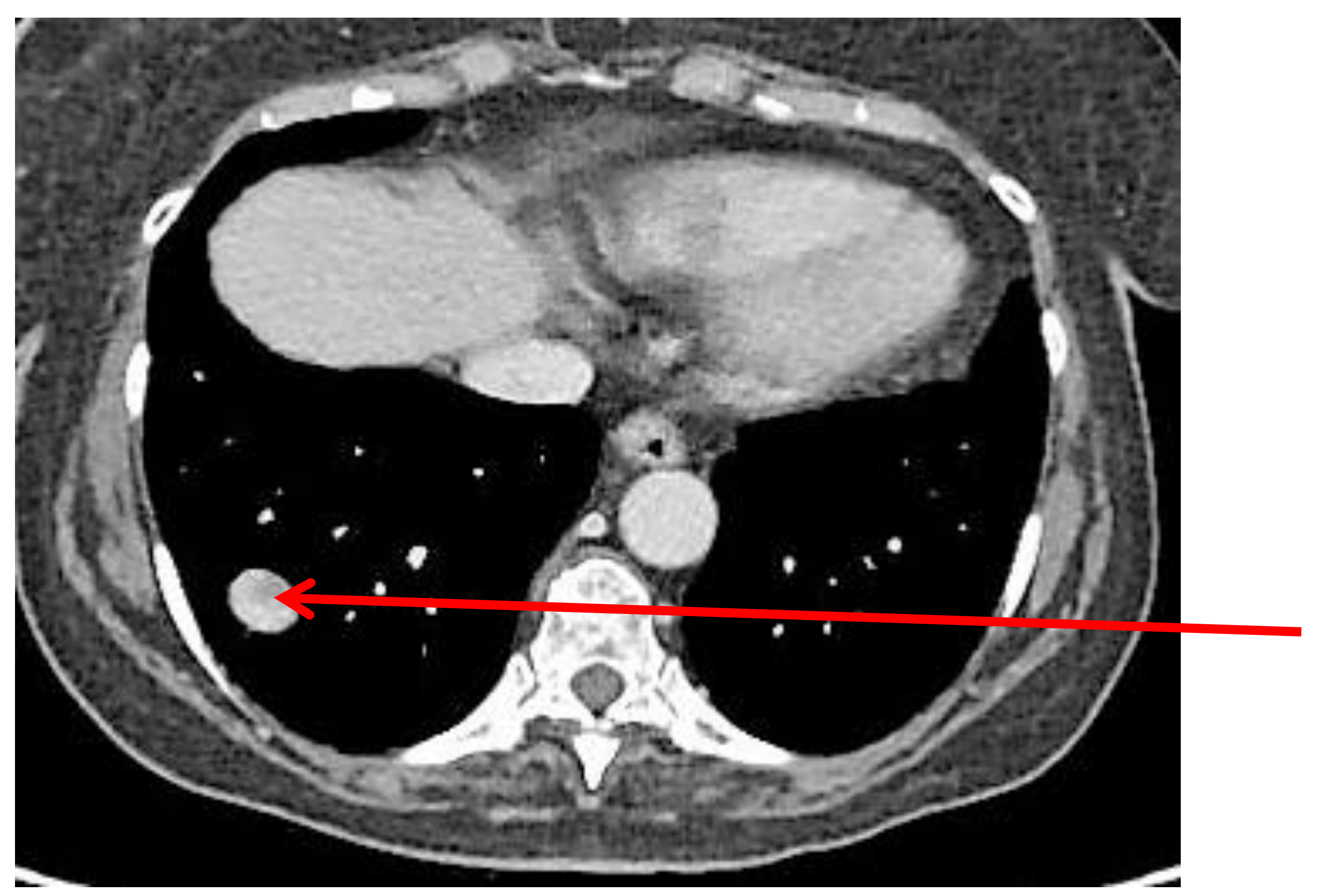

Pulmonary metastases

\section{Conclusion}

- ACC are rapidly progressive and aggressive. Our patient developed complications of hypercortisolaemia i.e. sigmoid bowel perforation, femoral vein thrombosis, severe hypokalaemia and poor wound healing. 